\title{
Placement of measurements for electrical distribution network state estimation
}

\author{
Irina Golub $^{1 *}$, and Yana Kuzkina ${ }^{2}$ \\ ${ }^{11}$ Melentiev Energy Systems Institute of Siberian Branch of Russian Academy of Sciences, Pipeline \\ Energy Systems Department, Lermontov str., 130, Irkutsk, Russia, 664033 \\ ${ }^{2}$ LLC "Engineering Center "Irkutskenergo", Irkutsk, Russia
}

\begin{abstract}
The paper is concerned with the problem of placement of the minimum number of smart meters to ensure either the observability of all state variables in distribution network or the observability of voltage magnitudes. Voltage control is important in the distribution network with distributed generation sources which adoption can lead to unpredictable overvoltage exceeding admissible values. The algorithm for smart meters including measurements of voltage magnitudes and active and reactive current injections is similar to the algorithm of choosing the minimum number of phasor measurement units to ensure topological observability. Optimal control of the active distribution network operation requires monitoring to be based on a classical linear state estimation procedure. The results of the research demonstrate the effectiveness of the proposed approaches and are illustrated by example of a test distribution network.
\end{abstract}

\section{Introduction}

The enhancement of the energy efficiency can be achieved through the creation of intelligent networks including electric and pipeline distribution networks [1]. The main feature of active intelligent networks is the integration of a distribution network with a communication network and a state-of-the-art measurement system. Such integration became possible due to the development of new information and communication technologies and the creation of Advanced Metering Infrastructure (AMI) [2]. The most important AMI components are smart meters (SM) installed at load nodes. Their data are transferred to a utility or a dispatcher, thus providing a two-way data exchange between a meter, a central system and a consumer. Consumers knowing their energy consumption and prices can manage their energy demand which in turn can reduce the total energy consumption.

Intelligent systems have been widely implemented not only in the electric power industry, but also there is AMI for collection and transmission of information about water, gas, and heat consumption. In other words, electro-SM, water-SM, heat-SM, gas-SM [3, 4] apply the same devices for information transfer and the same approaches to storage and processing results of measurements. Moreover, the group of EU Commission experts considers it is necessary to create integrated intelligent networks in which electrical and pipeline networks will be linked together [4]. EU is planning to replace $80 \%$ of all types of

* Corresponding author: golub@isem.irk.ru 
electric measurements in distribution networks by SM measurements by 2020 [5], the leaders in the installation of heat-SMs are Denmark, Sweden and China, and gas-SM - the UK and Italy [4].

Various communication tools can be used to create AMI, but the power line communication (PLC) technology found the widest application for electrical distribution networks. It provides high-speed data transfer throughout the power networks. Apart from the information about electricity consumption, SMs of some manufacturers can measure voltage magnitude, active and reactive power or current injections. As an example of such a SM we can consider a three-phase electronic device A1500. The device has a time marker with a measurement error not more than $0.5 \%$ and a 15 -minute interval between measurements [6]. The voltage information is important for power quality control at certain nodes, meanwhile full synchronized information about current loads and voltages is used for state estimation of the distribution network.

However, currently there is no standard for transfer of SM measurements, and the studies on the problem of applying SMs in the distribution network state estimation do not provide an unambiguous answer to the question about the possibility of their synchronization. $[2,6,7]$ Thus, the studies on the development of technologies that provide both phasor measurements in distribution network and their synchronization are conducted, and it is necessary to be ready to use them. In the analysis of algorithms for placing SMs in the distribution network, which are the focus of the paper, firstly SMs are supposed to measure phasors of current injections and voltage phasors, and then it is shown how the replacement of voltage phasor measurements with the measurements of voltage magnitudes affects the placement of SMs.

General approaches of the AMI organization for electrical and pipeline distribution networks allow to use similar algorithms for the placement of SMs. In the future, indications of such SMs will be used not only for energy consumption control, but also for the state estimation of the relevant pipeline networks

\section{Mathematical statement}

Optimal control of the active distribution network operation requires monitoring to be based on a classical linear state estimation procedure involving synchronized PMU (phasor measurement unit) measurements [9].

State estimation on the basis of PMU measurements suggests determining components of the state vector $x$, which, in an algebraic form of complex values, includes real $U_{a}$ and imaginary $U_{r}$ voltage components at the nodes of the calculated electrical network scheme on the basis of synchronized phasor measurements $z$, including measurements of voltage phasors $U \angle \delta_{U}=U_{a}+j U_{r}$ at nodes with PMU and phasors of currents $I \angle \delta_{I}=I_{a}+j I_{r}$ in the ties adjacent to such nodes. Measurements $z$ are related to the estimated variables of the state vector $x$ by the Jacobian matrix $H$. The elements of this matrix are parameters of the electrical network equivalent circuit; such a relation is linear

$$
z=H \cdot x
$$

Equality of the rank of a matrix $H$ to the number of state variables $x$ is a necessary condition for observability, which determines the existence of a solution to problem (1). Since the rank of the matrix $H$ largely depends on the network topology, the set and allocation of measurements, such observability is called topological.

With the PMU measurements used, node $i$ will be topologically observable, if voltage phasor is measured at this node, or voltage phasor at node $i$ can be determined by the measurements of voltage phasor at the adjacent node $j$ and current phasor in the tie line $i-j$. 
Additional information used to provide the observability is the information on equality of current injections to zero at transit nodes, i.e. at the nodes without generation and load. If at the transit node $i$ voltage phasor is known, then zero injection at node $i$ can be used to provide observability of any node $j$ adjacent to node $i$. The zero-injection node $i$ will be observable if voltage at any node $j$ adjacent to it is known.

In the distribution network the synchronized PMU measurements are not applied due to their high price [3]. Nevertheless, to place SMs in the distribution network we can apply the same approaches as to place the minimum number of PMUs [10] with the use of an integer linear programming procedure:

$$
\begin{aligned}
& \min _{x} f^{T}, \\
& A x \geq b
\end{aligned}
$$

where $f$ - vector of linear objective function coefficients, $b$ - vector of right-hand sides for inequality constraints, $A$ - a matrix, $x$ - a binary integer vector which elements take values 0 or 1, the latter indicate which nodes should be set to PMU. A detailed study of the algorithm for choosing the optimal composition of measurements, including when limiting the number of channels for current measurements and zero current injections is given in [9-11]. The problem of choosing the minimum number of PMUs is solved for the normal operation condition of the network (Task 1), for the condition of change in the network topology when some individual ties are disconnected, (Task 2), when some PMUs fail (Task 3), and for the case where some of the voltage or current phasor measurements that belong to the PMU are lost (Task 4).

\section{An example of a test distribution network}

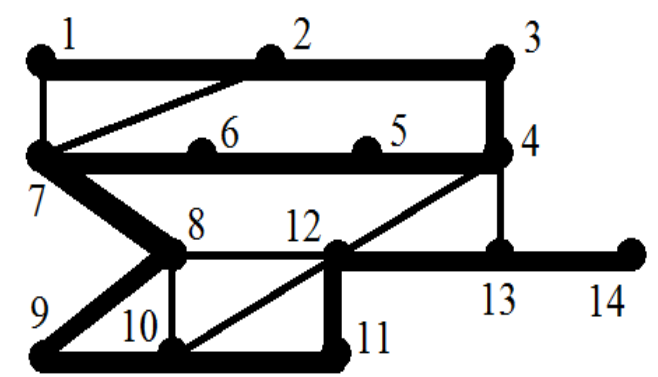

Fig.1. A 14-node IEEE test scheme with 20 ties, with the Hamiltonian path highlighted with a bold line

For the 14-node IEEE test scheme with 20 ties, Fig.1, we can show that the placement of one- or two-channel PMUs at it for Tasks 1-4 can be obtained when placing the PMUs at the nodes of the Hamiltonian path [12]. This way is demonstrated in Fig. 1 by a bold line, it passes through nodes 1-14 entering each of them only once.

The placement one- and two-channel PMUs at the nodes of Hamiltonian path for Tasks 1 and 3 is demonstrated in Fig.2 and Fig.3. The number of one-channel PMUs for these Tasks equals 7 and 14 and the number of two-channel PMUs -5 and 10, respectively.

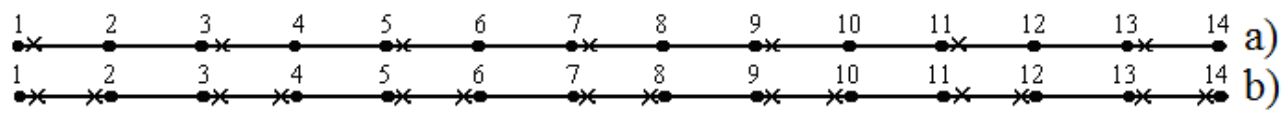

Fig.2. Sets of one-channel PMUs for the network in Fig.1, including phasor measurements of current $*$ and voltage $*$ for Task 1 (a) and Task 3 (b) 
The Hamiltonian path has a configuration typical for a low-voltage feeder without branching. Therefore, the solutions presented in Fig.2 and Fig.3 can be considered as optimal sets of PMUs providing solutions to Tasks 1 and 3 for the distribution network feeder.

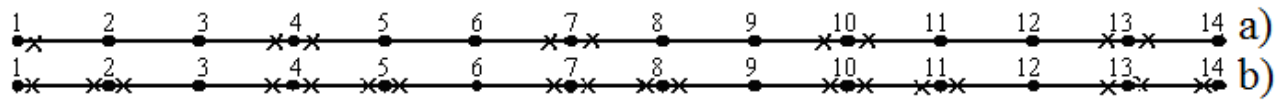

Fig.3. Sets of two-channel PMUs ***, for the network in Fig.2, including phasor measurements of current $*$ and voltage $*$ for Task 1 (a) and Task 3 (b)

\section{An optimal set of SMs in the distribution network on the basis of PMU placement results}

An analysis of possible selection of an optimal SM set by replacement of current measurements in feeder ties, Fig.2 and Fig.3, by the measurements of current injections shows that for Task 1 the optimal set of SM measurements can be obtained on the basis of placement of one-channel PMU, Fig.2a, for Task 3 - on the basis of placement of a twochannel PMU, Fig.3b. Such optimal sets of SMs are demonstrated in Fig.4.

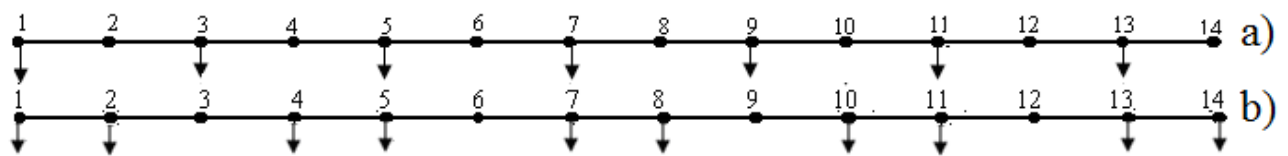

Fig.4. Sets of SM measurements for a 14-node distribution network without branches, including phasor measurements of current injections $\downarrow$ and voltage - for Task 1 (a) and Task 3 (b)

As previously noted, it is possible to synchronize measurements of active and reactive currents and voltage magnitudes for the SMs applied currently. We will show how the replacement of phasor measurements of voltage with the measurements of voltage magnitudes affects the selection of a set of SMs that provides the distribution network observability.

The $0.4 \mathrm{kV}$ low-voltage distribution network connected directly to consumers is characterized by a comparatively small inductance and a load with a high power factor. Therefore, the imaginary voltage component in the distribution network is considerably smaller than the real component. Experiments show that voltage phases do not exceed two degrees. This allows the state estimation of distribution network specify the measurements of real voltage components to be equal to the measurements of voltage magnitudes, and assume the measurements of imaginary voltage components to be equal to zero.

In the case where SMs with measurements of voltage magnitudes are installed at all load nodes of $N$-node network, the minimum number of measurements required to ensure observability should include $2(\mathrm{~N}-1)$ measurements of active and reactive current injections, a measurement of voltage magnitude at one of the nodes and a fixed voltage phase at the base node. The remaining $(N-2)$ measurements of voltage magnitudes will be redundant and can be used to increase the accuracy of the obtained estimates.

The diagrams presented in Fig.5 make it possible to compare the values of real voltage component for testing conditions of the 14-node low voltage feeder with the real voltage components that were obtained from linear state estimation for three sets of SMs.

The values of current injections and voltage magnitudes that were assumed to be equal to the measurements of real voltage components were obtained for the testing conditions 
and used as measurements for state estimation. The measurements of imaginary voltage components were set to be equal to zero.

The first set included SM measurements at nodes 2-14, the second one corresponds to Fig.4b (Task 3) and the third set corresponds to Fig.4a (Task 1). An analysis of the diagrams in Fig.5 shows that for all sets of SM measurements the real voltage components can be applied to control voltage levels at all the nodes of low-voltage feeder that is one of the main tasks in the distribution network with distributed generation.

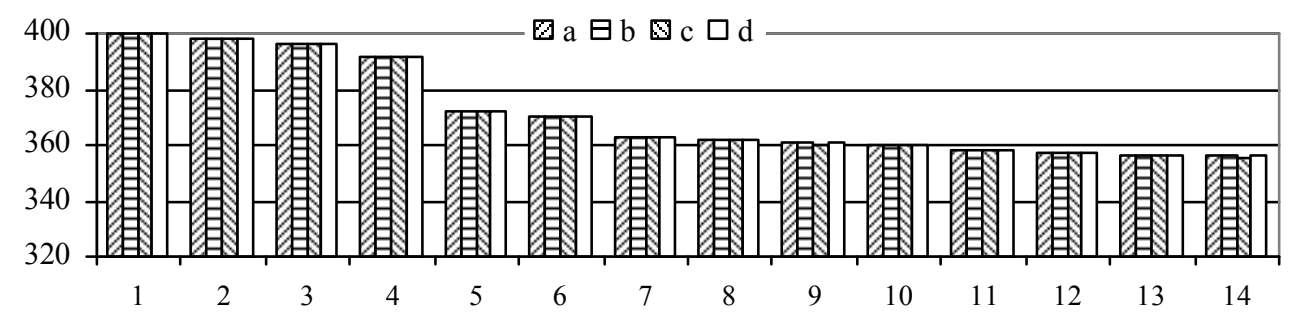

Fig.5. Values of real voltage components at nodes of a 14-node feeder: a - for the testing conditions, $\mathrm{b}-$ when SMs are installed at nodes 2-14, c - when SMs are installed according to Fig. $4 \mathrm{~b}, \mathrm{~d}$ - when SMs are installed according to Fig.4a.

The error in equating zero "measurements" of the imaginary voltage components leads to significant errors in the estimation of not only reactive but also active components of the currents in the branches and active current injections, as follows from the diagrams in Fig. 6 and Fig.7. Thus because of errors in the estimates of currents the observability of all state variables can be provided only when SMs are installed at all load nodes.

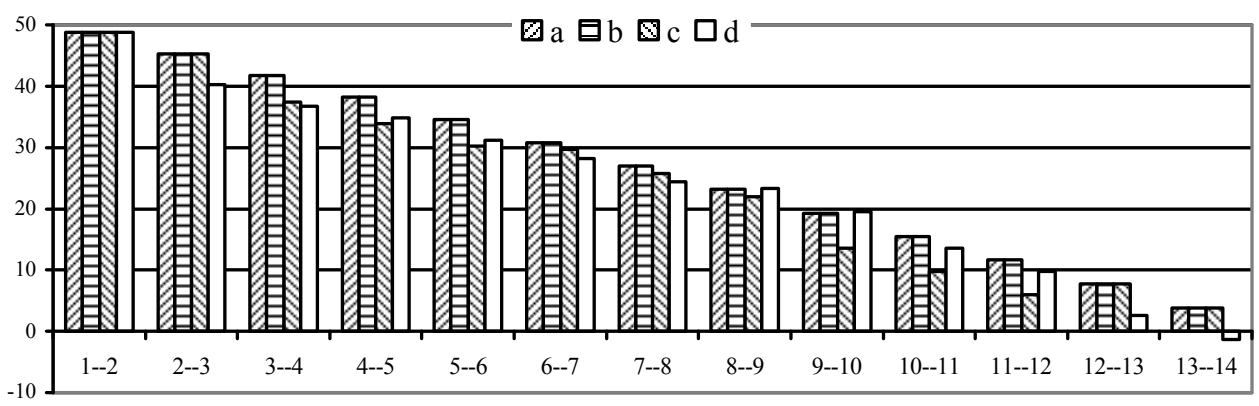

Fig.6. The values of the active components of the currents in branches of the 14th node feeder: a - for the test mode, b - when installing SM in nodes 2-14, c - when installing SM in accordance with Fig.4b, $d$ - when installing SM in accordance with Fig.4a.

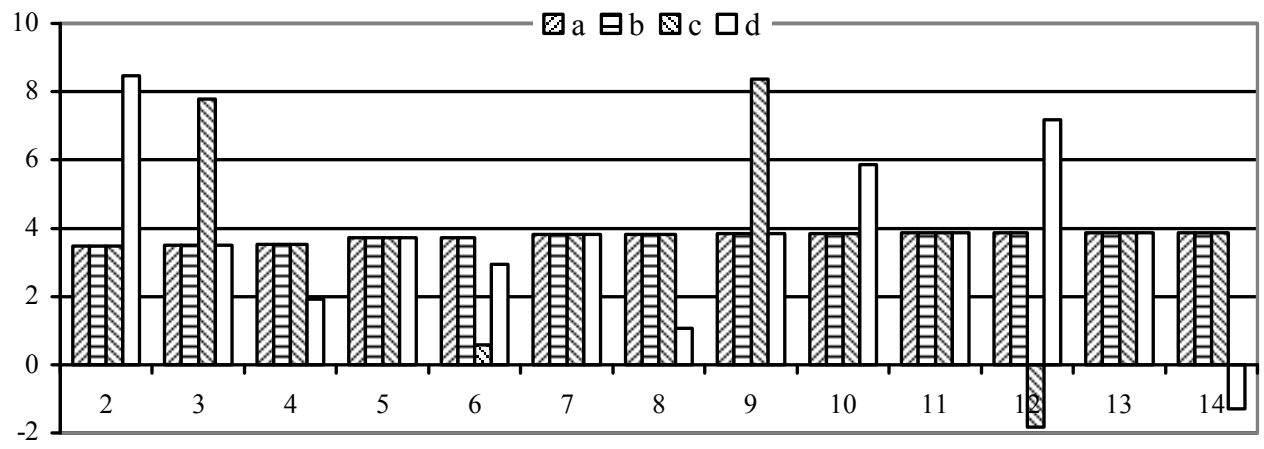

Fig.7. The values of the active current injections of the 14th node feeder: $a$ - for the test mode, $b$ - 
when installing SM in nodes 2-14, c - when installing SM in accordance with Fig. 5b, d - when installing SM in accordance with Fig. 5a.

\section{Conclusions}

1. It is proposed to use the algorithms for selecting the minimum number of PMUs in different operating conditions for the choice of the number and placement of SM measurements in the distribution network.

2. The minimum number of SM measurements for the normal operation condition of the network and when some SM measurements fail can be obtained by selection of one-channel and two-channel PMUs.

3. Replacement of phasor measurements of voltage in the sets of SM measurements with the measurements of voltage magnitudes makes it possible to provide observability real voltage components at all nodes of the distribution network.

4. Observability of all variables in a low-voltage distribution network can be provided only when SMs are installed at all load nodes.

5. Approaches similar to those proposed can be used to form SM formulations as separate pipeline distribution networks and to combine them with electric distribution networks.

The work is done in the framework of the project III.17.4.2. program of fundamental research SB RAS, registration number AAAA-A17-117030310438-1.

\section{References}

1. R.Wennersten, Q. Sun, H. Li, J CLEAN PROD, 103, 13 (2015)

2. R.R. Mohassel, A. Fung, F. Mohammadi, K. Raahemifar, CCECE, 8 (2014)

3. S. Santos, A Llano, A. Arzuaga, T.Arzuaga, L.Marron, M.Zamalloa, CIGRE, 8 (2012)

4. Q. Sun, H Li., Z. Ma, C. Wang, J. Campillo, Q. Zhang, F. Wallin, J. Guo. IEEE Io Journal. 3, 16 (2016)

5. Q. Chen, D. Kaleshi, Zh. Fan. IEEE ISGT EUROPE. 5 (2013)

6. W.H. Wellssow, D.Waeresch, PSCC. 7 (2014)

7. S. Rohrenbeck, IEEE PESS. 6 (2015)

8. Abart, D. Burnier, A. Lugmaier, CIRED. 4 (2011)

9. R.F. Nuqui, A.G Phadke, IEEE Power Tech. 4 (2007)

10. I.I. Golub, M.V. Khokhlov. Elektrichestvo 1, 8 (2015)

11. N. Fan, J-P Watson, Energy Systems, 1, 19 (2014)

12. Dharwadker, The Hamiltonian circuit algorithm (Amazon, 2011) 